\title{
Complex network analysis of the Brazilian power grid
}

\author{
Análise da rede complexa de distribuição de energia elétrica brasileira \\ G. C. Martins ${ }^{1}$; L. S. Oliveira ${ }^{1}$; F. L. Ribeiro ${ }^{2}$; F. L. Forgerini ${ }^{1 *}$ \\ ${ }^{1}$ Campus Paulo Freire, Universidade Federal do Sul da Bahia - UFSB, 45988-058, Teixeira de Freitas - BA, Brasil \\ ${ }^{2}$ Departamento de Física, Universidade Federal de Lavras - UFLA, 37200-000, Lavras - MG, Brasil
}

*fabricio.forgerini@ufsb.edu.br

(Recebido em 09 de junho de 2018; aceito em 19 de outubro de 2018)

\begin{abstract}
In the last decades, power grids and other energy transmission networks have received attention from the network literature. In this work, we studied one of the most important static features of power grids, their topology, with focus on the Brazilian Power Grid (BPG). We obtained the spatial structure of the BPG from the ONS (Electric System National Operator), consisting of its high-voltage transmission lines, electric stations and substations. Some traits had to be ignored, such as the local low-voltage substations and as well the dynamic features of the network. We analyzed the complex network of the BPG and its statistics, such as the mean degree, the degree distribution, the network size and the clustering coefficient to characterize the complex network. We also detected critical locations in the network and, therefore, points that are more susceptible to cascading failures and even to blackouts. Our results show that the BPG is functional against random failures, regarding random removal of links and connections, in terms of the size of the largest cluster. We observe that when a fraction $\rho$ of the links are randomly removed, the network may break down into smaller, disconnected parts. However, the largest component of the power grid remains connected. We believe that even a static study of the network topology can help to identify the critical situations and also prevent failures and possible blackouts.
\end{abstract}

Keywords: Power Grid, complex systems, complex networks

Nas últimas décadas, as redes de distribuição de energia elétrica e outras redes de transmissão têm recebido atenção da literatura de redes complexas. Neste trabalho, estudamos uma das mais importantes características estáticas de redes de energia, sua topologia, com foco na Rede Brasileira de Energia Elétrica (RBEL). Obtivemos a estrutura espacial da RBEL do Operador Nacional do Sistema Elétrico (ONS), composto por linhas de transmissão de alta tensão, estações elétricas e subestações. Algumas características tiveram que ser ignoradas, como as subestações locais de baixa tensão além dos recursos dinâmicos da rede. Analisamos a rede brasileira e suas estatísticas, como o grau médio, a distribuição de graus, o tamanho da rede e o coeficiente de agrupamento para caracterizar a rede complexa. Também detectamos locais críticos na rede e, portanto, pontos mais suscetíveis a falhas que podem gerar defeitos em cascata e até a apagões. Nossos resultados mostram que a RBEL se mantem funcional contra falhas aleatórias, quanto à remoção aleatória de links e conexões, quando analisado em termos do tamanho do maior componente da rede. Observamos que quando uma fração $\rho$ dos links é removida aleatoriamente, a rede pode se dividir em partes menores e desconectadas. No entanto, o maior componente da rede elétrica permanece conectado. Acreditamos que este estudo estático da topologia da rede pode ajudar a identificar as situações críticas e também evitar falhas e possíveis apagões.

Palavras-chave: Rede de Energia Elétrica, Sistemas Complexos, Redes Complexas

\section{INTRODUCTION}

Power grids are part of what have been called technological networks, the physical infrastructure network that transports of energy and information [1]. The Internet and telephone networks are some examples of these type of networks. This field has received attention from researchers and the correspondent literature in the last decades mainly due to problems caused by the possibility of large scale failures, that could lead to major blackouts $[2,3,4,5]$. Despite the fact that power grids dynamics have been managed by computer systems and human operators [6], the static features of this type of network can be studied to provide valuable information for private or public investments and preventing failures [7], and one of the most important static features is the topology of a network. 
We studied the topology of the Brazilian Power Grid (BPG) in this work. We obtained the spatial structure of the BPG from the Electric System National Operator (ONS), which consists of the high voltage transmission lines, generator stations and substations. The local low-voltage substations were neglected in this work. We also neglected the dynamic features of the power grid, such as the electromagnetic processes and human interference by controlling the energy flux on the network.

We analyzed the BPG as a complex network in which all stations and substations were represented by nodes, and transmission lines were represented by links among the nodes. By identifying the network characteristics, we use them to find out its weakness and thus we can suggest improvements in order to prevent failures and blackouts. Our work show that the connected component of the network rapidly decays with the random removal of links and nodes, as the size of the largest cluster smoothly decay, which is common to sparse networks with a percolation threshold at finite $\rho_{\mathrm{c}}$.

This paper is organized as follows. In Sec. 2, we present our methodology and details of the data analysis. In Sec. 3, we describe our main results and discuss them. Finally, in Sec. 4, we summarize the results and present our main conclusions.

\section{METODOLOGY}

The BPG data was obtained from the ONS database, covering the latest update, from 2015. The network analysis was performed by using network theory, focusing on topological properties, namely the mean degree and the degree distribution, betweenness centrality, mean clustering coefficient and the connectivity of the network.

The degree $k_{i}$ is the number of links attached to a node $i$, in a total of $N$ nodes, given by the number of transmission lines connected to the station or substation. On the other hand, the mean degree of the network is a global measure, given by

$$
\left\langle k_{i}\right\rangle=\frac{1}{N} \sum_{i=1}^{N} k_{i} .
$$

The degree distribution $P(k)$ is the probability that a node chosen uniformly at random within the network has a degree $k$ :

$$
P(k)=\frac{\langle N(k)\rangle}{N}
$$

where $N$ is the total number of nodes in the network and $\langle N(k)\rangle$ is the average number (or fraction) of nodes with degree $k$ on the network [3].

The distribution is given by the equation 2 and, once it is known, we can obtain information by calculating the moments of this distribution. The $n$-th moment of the distribution is

$$
\left\langle k^{n}\right\rangle=\sum_{k=0}^{\infty} k^{n} P(k)
$$

The first moment $\langle k\rangle$ is the mean degree, while the second moment is a measure of the degree fluctuation of the distribution. If $\left\langle k^{2}\right\rangle$ diverges, the structure and function dramatically change in the network, in contrast to those with finite $\langle k\rangle[8]$.

An exponential degree distribution has the form

$$
P(k)=C e^{-\alpha k}
$$

where $C$ is an arbitrary constant. Networks with exponential degree distributions were reported to follow this pattern in some real-world situations, such as power grids [9]. The clustering 
coefficient of the entire network, or the mean clustering coefficient, is the average of the local clustering coefficient over all nodes:

$$
\bar{c}=\sum_{k} P(k) \bar{c}(k)
$$

and may vary between 0 and 1 . The betweenness centrality is a measure of centrality in which we quantify the extent to which a node lies on paths between other nodes and is proportional to the number of paths of each node on the network [1]. In other words, nodes with high betweenness centrality may have a larger influence on the network by the reason of their importance controlling the flow passing between other nodes. Matematically, let $n_{a b}^{i}$ be 1if node $i$ lies on the path between $a$ to $b$ and 0 if does not (or there is no path between $a$ to $b$ ) and the let $g_{a b}$ to be the total number of paths from $a$ to $b$. Then the betweenness centrality $x_{i}$ is given by

$$
x_{i}=\sum_{a b} \frac{n_{a b}^{i}}{g_{a b}}
$$

We can also visualize the network structure with the corresponding positions of the generator stations and substations and the transmission lines connecting them. As one can see in Figure 1, we show the Brazilian Power Grid and its corresponding complex network. This picture was created by using the large network analysis software Pajek ${ }^{\mathrm{TM}}[10]$.
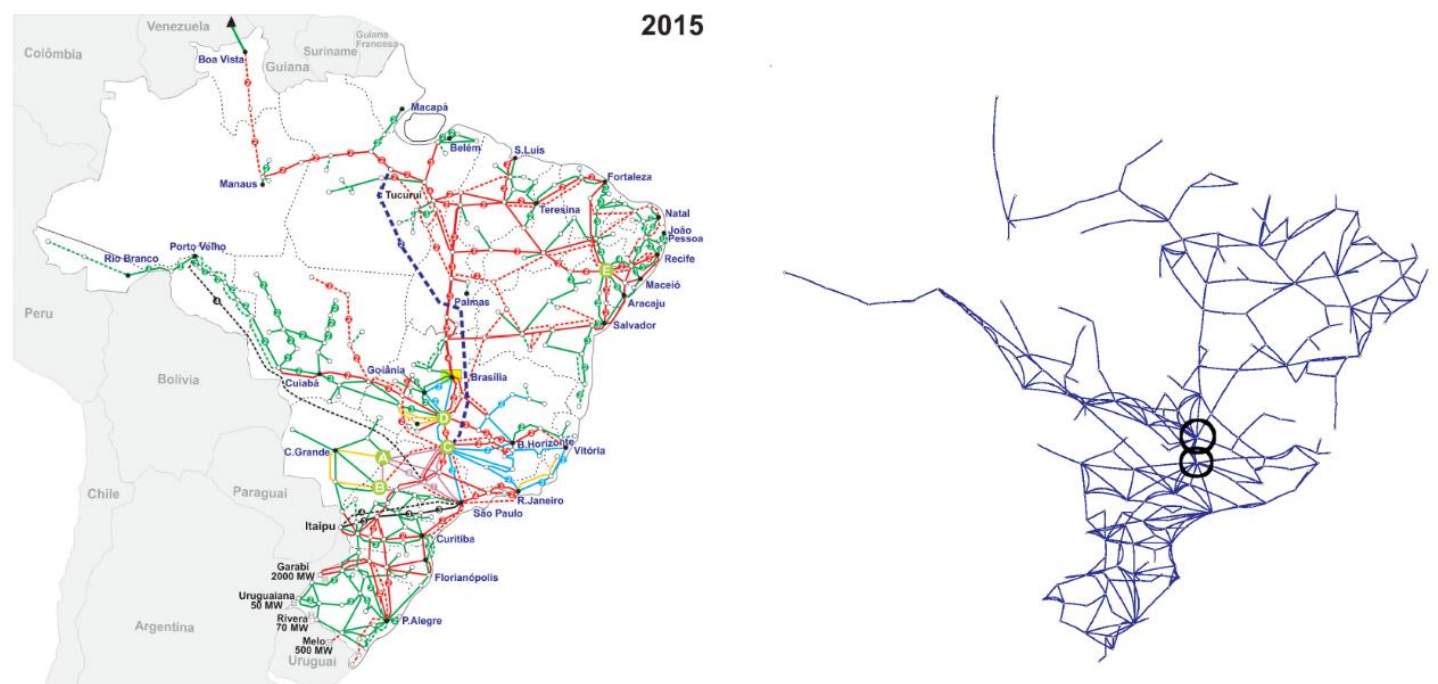

Figure 1: The Brazilian Power Grid (left panel) and its representation as a network (right panel). The traced lines at the left panel represent the transmission lines under construction and were not considered in this work, as one can see at the right panel. We also show, inside the black circles, important nodes that keep the network connected, as we will discuss in section III.

As mentioned before, in this work we do not consider electromagnetic processes, computer control and human interference in the energy flux of the network. Despite the fact that we neglect the dynamic features of the network, our analysis might not be so naïve after all. As we will show in next section, the BPG is strongly dependent on the topological structure. In case of large structural failures, failures propagating through cascade effects may not be corrected by human interference or computer systems. We believe that the this approach can provide relevant information about the BPG's vulnerability.

\section{RESULTS AND DISCUSSION}

The BPG has developed in the last decades, but so far it is much smaller in comparison to other power grids worldwide. For example, the USA's power grid is represented by a network of 14099 
nodes and 19657 links [9]. The main metric results of the characterization of the BPG are shown in Table 1. One can see that these measures represent the most basic topological information of the network.

Table 1: Main results of the topological analysis of the Brazilian Power Grid and its size compared with the US Power Grid [6, 9].

\begin{tabular}{ccc}
\hline Characteristics & Brazil & USA \\
\hline Mean Degree & 6.487 & 1.394 \\
Number of nodes & 230 & 14099 \\
Number of links & 1492 & 19657 \\
Clustering coefficient & 0.174 & 0.080 \\
Betweenness Centrality & 0.438 & $\sim 0.642$ \\
\hline
\end{tabular}

The degree distribution is an important network measure. Much information about a network is related to the degree distribution. Networks with heavy tail scale-free degree distribution have been studied over a decade. A network with a power-law degree distribution $P(k) k^{-\gamma}$ with $2 \leq$ $\gamma \leq 3$, for instance, is expected to be resilient to random removal of links, i.e. resilient against random failures of the grid [11]. Numerous real world networks, including the Internet, have a power-law degree distribution with $\gamma \approx 3$ and in all of them the resilience phenomena was observed $[12,13]$. For cases where $\gamma>4$, this type of networks are known as ultra resilient against random failures [1].

In this work we use the cumulative degree distribution,

$$
P(K>k)=\sum_{k=K}^{N} P(k)
$$

which is the probability that the degree is greater or equal to $\mathrm{k}$. By using the cumulative degree distribution to represent the data, we reduce the noise in the tail of the distribution. As one can see in Figure 2, the cumulative degree distribution is shown and it has the exponential form.

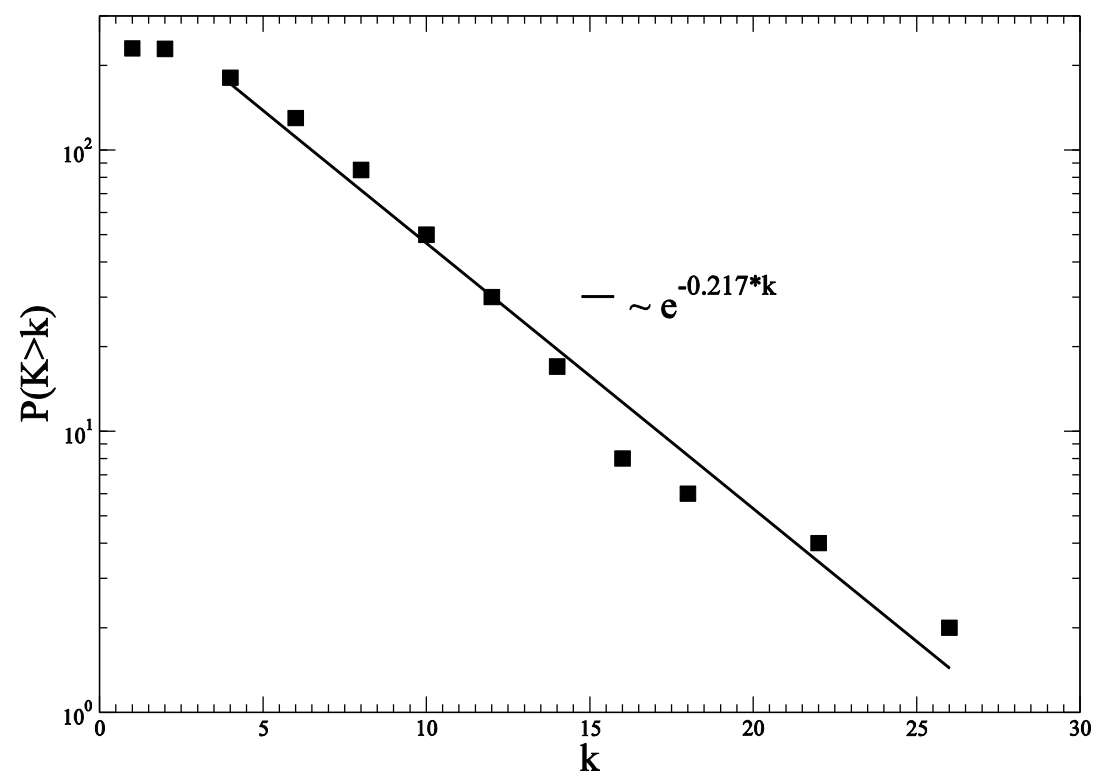

Figure 2: Cumulative degree distribution, $P(K>k)$, of the Brazilian Power Grid. As shown in the picture of semilogarithmic scale, the degree distribution has an exponential form.

Another important measure is the mean clustering coefficient of the network $c$. The concept of clustering reflects how the first neighbors of a node are connected to each other. This feature is a statistic of the number of multiple connections among the nodes in the network. In other words, clustering coefficient refers to the statistics of the number of triangles (loops of length 3 ) in the network. Regarding to the mean clustering coefficient of the BPG, we found $\bar{c}=0.174$, a 
very small value for the clustering coefficient. This indicate that the BPG is, in average, poorly connected and highly susceptible to formation of isolated clusters in the network.

In order to investigate the connectivity of the network and how random removal of links and nodes affect it, we studied the number of connected components of the network, $C$, and also the size of the largest cluster of the network, $S$. As one can see by our simulations, both measurements of connectivity presents different but complementary results.

For the first simulation, when all the links are present, the fraction of removed links, $\rho$, is equal zero and the network is a single connected component; for this case, $1 / C=1$. As the number of removed links increases, the network is divided into disconnected parts and $1 / C \rightarrow 0$. When the fraction of removed links is $\rho=1,1 / C=0$. We performed simulations to investigate the network connectivity. By removing randomly links from the network, we calculate the number of connected components for different scenarios. Our results are shown in the Figure 3, where one can see on the left axis the plot $1 / C$ versus $\rho$. We show that for a small fraction of links removed, $\rho \approx 0.1$, the Brazilian Power Grid turns into a set of several disconnected parts (small value of $1 / C)$. The power grid rupture is significantly important for the Brazilian case, since the number of generator stations is relatively small.

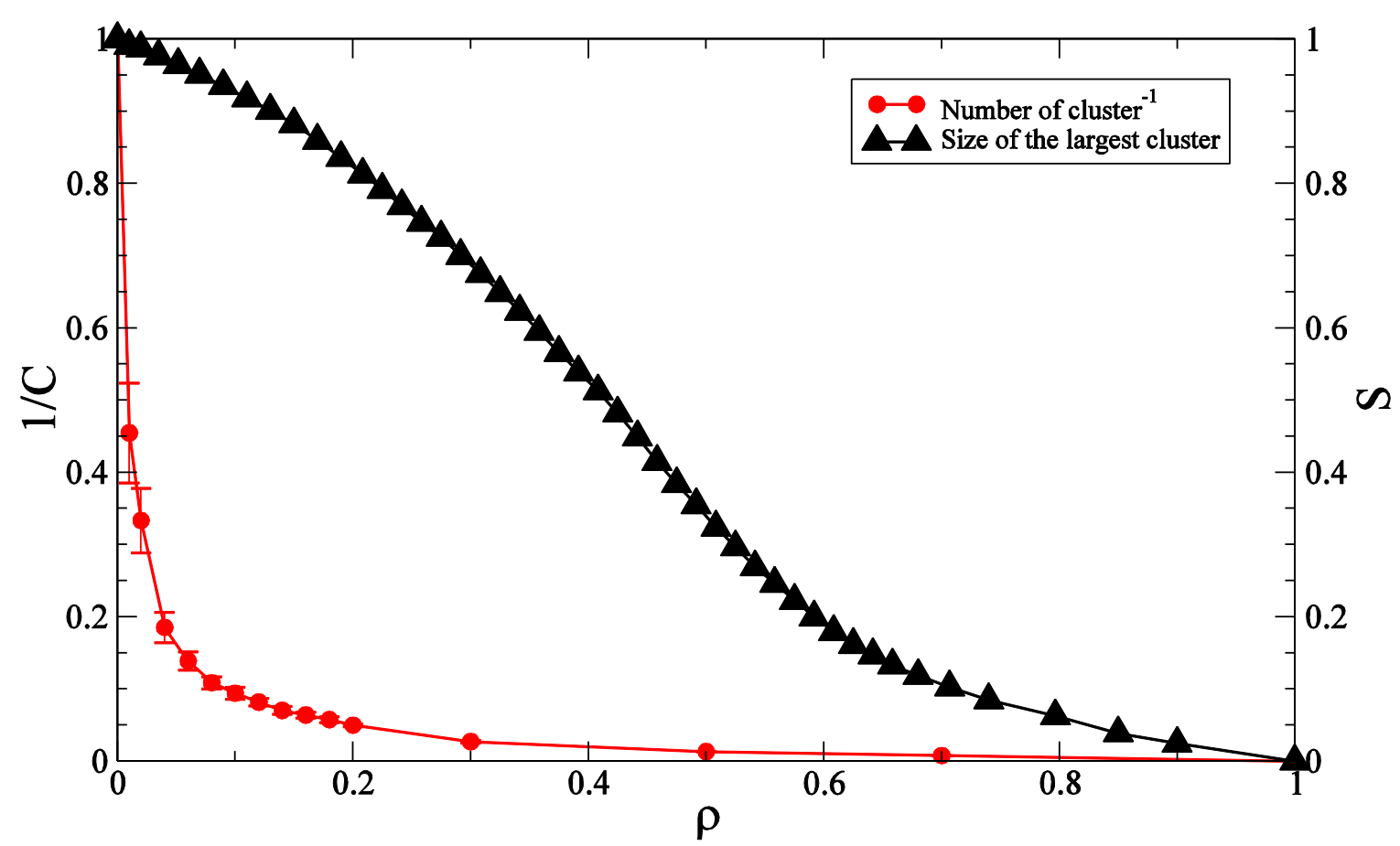

Figure 3: The number of connected components of the network, $C$, and the size of the largest cluster on the network, $S$. In the graph, we show in the left axis the $1 / C$ versus $\rho$ and, in the right axis, $S$ versus $\rho$. The results were obtained by averaging 100 different simulations. This result suggests that even if the network turn into a set of disconnected parts, for small $\rho$, the Brazilian Power Grid keeps the largest component connected.

The second connectivity measurement that was studied is $\mathrm{S}$. As one can see on the right axis of Figure 3, the decaying of $\mathrm{S}$ is not as fast as $1 / C$. For small values of $\rho, S$ is almost linear, following by an exponential regime. As shown, even for small values of $\rho$, the network turn into a set of several disconnected parts. However, most of these disconnected parts are isolated nodes, not affecting substantially the connected component of the network. Only for larger values of $\rho$ the size of largest cluster $S$ shows a rapidly decreasing. This result show that the topological structure of the Brazilian Power Grid proves itself, in some sense, functional against random failures.

We also made simulations considering nodes instead of links. Differently from our previous results, in the data shown in Figure 4 one can see a discontinuity in the size of the largest connected component, $\mathrm{S}$, which is evolving from a linear decaying to an exponential one. 


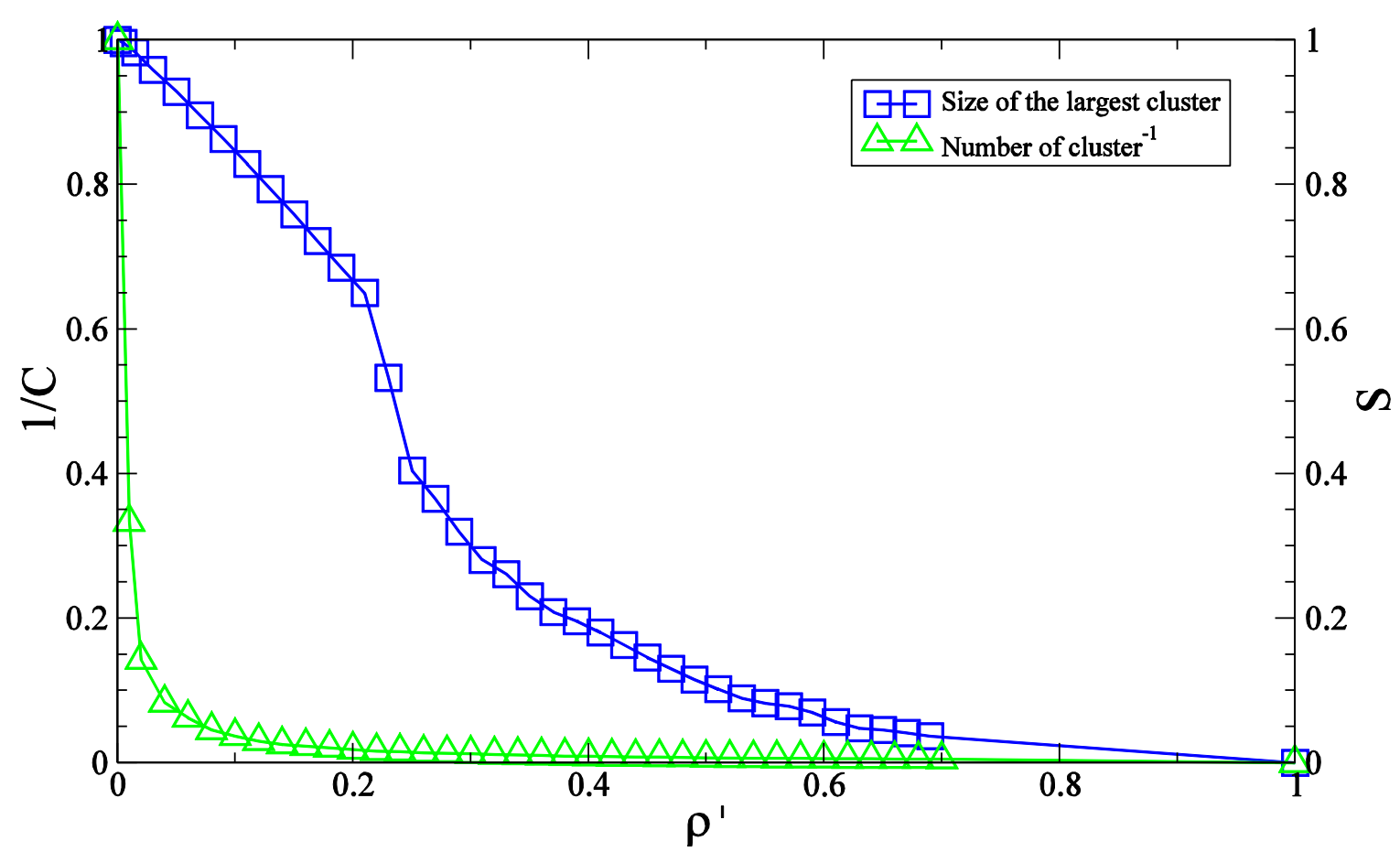

Figure 4: Network connectivity as function of the fraction of nodes removed at random from the network. Similarly to Figure 3, we measure the number of connected components on the network, $C$ and the size of the largest cluster on the network, $S$.

Analyzing the BPG one can see that there are few nodes playing an important role on keeping the network connected. The most important (and susceptible) nodes are shown in Figure 1 as two black circles. They both belong to a complex of hydroelectric power plants and their substations called Furnas Centrais Elétricas. These critical locations in the network connect the southsoutheast, the central part of the country and then the north and northeast regions. Failures in the nodes of this major electric complex will lead the BPG system to a cascading failure and even to major blackouts.

\section{CONCLUSION}

In this work we studied and analyzed the topology of the Brazilian Power Grid (BPG). Despite the fact that the BPG is comparatively smaller than power grids from other countries, by analyzing its spatial structure we show that the BPG is poorly connected and highly dependent on a few nodes to maintain the network's connected component. We summarize the most basic topological information of the BPG.

The degree distribution of the BPG has an exponential form and, according to the network literature, it is not resilient against random failures. We also simulate random failures on the network by removing links at random. Our results show that with a small fraction of missing connections, $(\rho \approx 0.1)$, the network turns into a set of several disconnected parts, leading parts of the country isolated. However, these isolated parts of the network do not represent the larger part of the power grid. We show that random removal of links does not affect significantly, for small values of $\rho$, the size of the largest cluster on the network, $S$, keeping the most of the country connected by the power grid.

In conclusion, one can see the main topological information and its weaknesses even through a static analysis of the network. In order to improve the network, to make it more resilient against random failures, the BPG needs improvements regarding the number of connections, increasing the mean shortest-paths to prevent failures and future blackouts. 


\section{ACKNOWLEDGMENTS}

The authors would like to thank CNPq and UFSB for the financial support (respectively, through project No. 409597/2016-3 and project 005/2015-PROSIS).

\section{REFERENCES}

1. Newman MEJ. Networks: an Introduction. Oxford: Oxford University Press; 2010, doi:10.1093/acprof:oso/9780199206650.001.0001

2. Dorogovtsev SN. Lectures on Complex Networks. Oxford: Oxford University Press; 2010, doi:10.1093/acprof:oso/9780199548927.001.0001

3. Forgerini FL. Interacting Agents and Stochastic Process on Complex Networks. Saarbrücken: LAP Lambert Academic Publishing; 2015.

4. Motter AE. Cascade control and defense in complex networks. Phys Rev Lett. 2004;93:098701, doi:10.1103/PhysRevLett.93.098701

5. Dobson I, Carreras BA, Lynch VE, Newman DE. Complex systems analysis of series of blackouts: cascading failure, critical points, and self-organization. Chaos. 2007;17:026103, doi:10.1063/1.2737822

6. Watts DJ, Strogatz SH. Collective dynamics of 'small-world' networks. Nature. 1998;393:440, doi: $10.1038 / 30918$

7. Arianos S, Bompard E, Carbone A, Xue F. Power grids vulnerability: a complex network approach. Chaos. 2009;19:013119, doi:10.1063/1.3077229

8. Boccaletti S, Latora V, Moreno Y, Chavez M, Hwang D-U. Complex networks: Structure and dynamics. Phys. Rep. 2006;424:175, doi:10.1016/j.physrep.2005.10.009

9. Albert R, Albert I, Nakarado GL. Structural vulnerability of the North American power grid. Phys Rev E. 2004;69:025103(R), doi:10.1103/PhysRevE.69.025103

10. Pajek project webpage: http://mrvar.fdv.uni-lj.si/pajek/

11. Cohen R, Erez K, ben-Avraham D, Havlin S. Resilience of the Internet to Random Breakdowns. Phys Rev Lett. 2000;85:4626, doi:10.1103/PhysRevLett.85.4626

12. Albert R, Jeong H, Barabsi A-L. Diameter of the world wide web. Nature. 1999;401:130, doi:10.1038/43601.

13. Albert R, Jeong H, Barabsi A-L. Attack and error tolerance of complex networks. Nature. 2000;406:130, doi:10.1038/35019019. 